\title{
Pneumatosis Intestinalis in COVID-19: Case Series
}

Kelvin Wong ${ }^{1}$, Dae Hyeon Kim ${ }^{1}$, Sameer Khanijo ${ }^{1}$, Aleksandr Melamud ${ }^{1}$, Gulrukh Zaidi ${ }^{1}$

1. Pulmonary and Critical Care Medicine, Northwell Health North Shore University Hospital/Long Island Jewish Medical Center, Manhasset, USA

Corresponding author: Kelvin Wong, kwong11@northwell.edu

\begin{abstract}
Objective: To describe the clinical characteristics and outcomes of patients with coronavirus disease 2019 (COVID-19) who developed pneumatosis intestinalis (PI).

Methods: This case series was conducted in intensive care units at two large tertiary care centers within the Northwell Health System, located in New York State. Patients were included if they were identified as having confirmed COVID-19 as well as pneumatosis intestinalis from March 16, 2020 to July 31, 2020. Patient demographics, clinical characteristics, vasopressor use, anticoagulation use, opiate use, paralytic use, COVID-19 treatment regimen, serum lactate, arterial pH, serum bicarbonate, subsequent intervention, and outcomes during hospitalization were collected.
\end{abstract}

Results: A total of nine patients were identified. Average serum lactate was $4.33 \mathrm{mmol} / \mathrm{L}$ at time of diagnosis. Portal venous gas (56\%) and bowel dilation (56\%) were common radiographic findings. Subsequent morbidity (increased vasopressor requirements - 67\%, acute kidney injury - 67\%, increased oxygen requirements $44 \%$ ) and mortality (78\%) were high. PI occurred despite a majority of patients being on anticoagulation (78\%). Interleukin-6 (IL-6) inhibitors were commonly administered (56\%) prior to development of PI.

Conclusion: Pneumatosis intestinalis in COVID-19 is clinically significant, with high morbidity and mortality, and is also likely underdiagnosed.

Review began 09/23/2020 Review ended 10/15/2020 Published 10/16/2020

() Copyright 2020 Wong et al. This is an open access article distributed under the terms of the Creative Commons Attribution License CC-BY 4.0., which permits unrestricted use, distribution, and reproduction in any medium, provided the original author and source are credited.
Categories: Gastroenterology, General Surgery, Infectious Disease

Keywords: covid-19, severe acute respiratory syndrome coronavirus 2, pneumatosis cystoides intestinalis, intestinal perforation, interleukin-6

\section{Introduction}

As of September 15, 2020, the number of confirmed coronavirus disease 2019 (COVID-19) cases have exceeded 29 million worldwide, resulting in almost one million deaths. As scientific and medical communities continue efforts to elucidate how the responsible pathogen, severe acute respiratory syndrome coronavirus 2 (SARS-CoV-2), can affect the human body, we have discovered many, often unexpected, ways it can cause harm.

Pneumatosis intestinalis (PI) refers to the presence of gas within the intestinal wall. Many aspects of this condition are poorly understood, including etiology, pathogenesis, and clinical implication. Reported etiologies have been widely varied, including gastroenteritis [1], chronic obstructive pulmonary disease, bowel obstruction, idiopathic [2], etc. While it may be a benign, incidental finding, it may also herald a lifethreatening condition such as intestinal ischemia. Previous studies have shown that elevated lactate, vasopressor use, small bowel dilation, peritonitis, and abdominal pain are predictive factors for clinically significant PI [3-5].

PI has been previously reported in COVID-19 patients, but it has been limited to three single case reports and a review of abdominal imaging findings in COVID-19 patients. In their review of 134 patients, Bhayana et al. reported that abdominal computed tomography (CT) detected pneumatosis or portal venous gas in four of 20 critically ill patients (20\%) [6]. In some of these patients, surgical findings showed unusual yellow discoloration of bowel and bowel infarction while pathology demonstrated ischemic enteritis, patchy necrosis, and fibrin arteriolar thrombi.

Here, we report a case series of PI in patients with COVID-19 infection and describe their clinical characteristics and outcomes.

\section{Materials And Methods}

Data was collected retrospectively from the electronic medical record (EMR), Enterprise Sunrise Clinical Manager-Allscripts, for patients with confirmed COVID-19 and pneumatosis intestinalis admitted to North 
Shore University Hospital and Long Island Jewish Medical Center, two large tertiary care centers within the Northwell Health System, from March 16, 2020 to July 15, 2020. This case series was approved by the Northwell Institutional Review Board (IRB), as minimal-risk research using data collected for routine clinical practice and waived the requirement for informed consent (IRB number 20-0200).

A confirmed case of COVID-19 was defined as a positive laboratory test for SARS-CoV-2 from real-time reverse transcription polymerase chain reaction (RT-PCR) assay of a respiratory specimen. Pneumatosis intestinalis was identified by abdominal CT. We collected data on patient demographics, comorbidities, time from symptom onset to PI diagnosis, CT imaging findings, concomitant lab values (serum lactate, arterial $\mathrm{pH}$, serum bicarbonate), subsequent intervention, COVID-19 treatment regimen, vasopressor use, anticoagulation use, opiate use, paralytic use, and patient outcomes (change in vasopressor requirements, change in oxygen requirements, development of acute kidney injury, resolution of PI and in-hospital mortality).

\section{Results}

A total of nine patients were included in our case series. The patients were predominantly male (seven patients, $77.8 \%$ ) with an average age of 54.3 years. Three patients were Hispanic (33.3\%) with the rest equally distributed among White, Black, and Asian ethnicities (two patients, 22.2\%). The most common comorbidity was obesity (six patients, 66.7\%), followed by hypertension (three patients, 33.3\%), diabetes (two patients, $22.2 \%$ ), and prior history of cerebrovascular accident or transient ischemic attack (two patients, $22.2 \%$ ). The average time of symptom onset to PI diagnosis was 22.2 days and the average time of admission to PI diagnosis was 13.7 days (Table 1 ). 


\section{Cureus}

Demographics

Total Cohort $(\mathrm{n}=9)$

Age (years)

Mean [Range]

Gender

Male

Female

BMI (kg/m2)

Mean [Range]

Normal (18.5 to < 25)

Overweight $(25$ to $<30)$

Obese $(>30)$

Race

Hispanic

White

Asian

Black

Comorbidities

HTN

DM

Prior CVA

CKD

Asthma

Medications at time of diagnosis

Anti-coagulation

Opiate

Vasopressor

Paralytic

COVID-19 Specific Medications

Steroids

Anti-II 6

Plasma

Plaquenil

Anti-II 1

Azithromycin
3 (33)

7 (78)

$54.3[36-65]$

7 (78)

2 (22)

32.8 [23.1 - 56.4]

2 (22)

2(22)

5 (56)

2 (22)

2 (22)

2 (22)

3 (33)

2 (22)

2 (22)

1 (11)

1 (11)

7 (78)

5 (56)

3 (33)

$6(67)$

5 (55)

3 (33)

3 (33)

2 (22)

2 (22)

\section{TABLE 1: Patient Demographics and Characteristics}

Note: Values are expressed as number (percentage) unless otherwise indicated 


\section{Cureus}

workup (four patients, 44.4\%). Other indications included workup for elevated serum lactate (two patients, $22.2 \%$ ), abdominal distension (two patients, $22.2 \%$ ), and confirmation of incidental findings on chest CT (one patient, 11.1\%).

Most patients had pneumatosis of both small and large bowel (six patients, 66.7\%), while two patients (22.2\%) had only small bowel involvement and one patient (11.1\%) had only large bowel involvement. Portal venous gas was present in five patients (55.5\%). Bowel dilation was also present in five patients (55.5\%), of which three patients (33.3\%) had dilated loops of both large and small bowel, while two patients (22.2\%) had dilated loops of small bowel alone (Table 2). 


\section{Cureus}

Clinical Data

Total Cohort $(n=9)$

Days from symptom onset to diagnosis of pneumatosis

Mean [Range]

$22.2[4-47]$

Days from admission to diagnosis of pneumatosis

Mean [Range]

$13.7[0-31]$

Laboratory Findings

Lactate (mmol/L)

Mean (SD)

Arterial pH

Mean (SD)

$7.22(0.13)$

Bicarbonate (mmol/L)

Mean (SD)

$19.33(5.83)$

Radiology Findings ( $n, \%)$

Portal Venous Gas

5 (56)

Dilated Bowel Loops

$5(56)$

Small Bowel Only

2 (22)

Large Bowel Only

$0(0)$

Both

Interventions (n,\%)

NPO

Antibiotics

7 (78)

Stopped Bowel Regimens

Total Parenteral Nutrition

Surgical intervention

Outcome (n,\%)

Increased Vasopressor Requirements

Acute Kidney Injury

$6(67)$

Inpatient Deaths

7 (78)

Discharged

2 (22)

Days from pneumatosis diagnosis to death

Mean [Range]

$6.43[0-16]$

\section{TABLE 2: Clinical Data}

At the time of CT diagnosis of PI, the average serum lactate was $4.33 \mathrm{mmol} / \mathrm{L}$ (range 0.8 to 12.4 ). Average arterial $\mathrm{pH}$ was collected for eight patients, with an average value of 7.22 (range 7.06 to 7.39 ). Average serum bicarbonate was $19.33 \mathrm{mmol} / \mathrm{L}$ (range 9 to 30) (Table 2).

The majority of patients were on full anticoagulation (seven patients, 77.8\%) with four patients on argatroban and three patients on unfractionated heparin. Most were also on continuous opiate infusions for sedation (seven patients, $77.8 \%$ ). Of the patients in our series, five (55.6\%) were on vasopressor medications. A minority of patients were on paralytics (three patients, 33.3\%) (Table 1). 
COVID-19-specific therapy included a variety of different regimens. The most commonly used medications were steroids (six patients, 66.7\%) followed by IL-6 inhibitors (five patients, 55.6\%), convalescent plasma (three patients, 33.3\%), hydroxychloroquine (three patients, 33.3\%), interleukin-1 inhibitors (two patients, $22.2 \%$ ) and azithromycin (two patients, 22.2\%). Two patients (22.2\%) received no COVID-19-specific therapy (Table 1).

After diagnosis of PI, one patient (11.1\%) underwent an exploratory laparotomy, which was done emergently at bedside and revealed necrotic bowel. All other patients were managed conservatively with bowel rest (100\%), empiric antibiotics to cover intestinal flora (77.8\%), and discontinuation of bowel regimens (33.3\%). Two patients (22.2\%) were also started on total parenteral nutrition (TPN) (Table 2).

Following diagnosis of PI, six patients (66.7\%) developed increased vasopressor requirements, six patients (66.7\%) developed acute kidney injury, and four patients (44.4\%) developed increased oxygen requirements. Resolution was demonstrated on interval imaging in two patients (22.2\%). In terms of mortality, seven patients expired (77.8\%) and two were discharged (22.2\%). For those who expired, the average time from PI diagnosis to death was 6.4 days (Table 2).

\section{Discussion}

To our knowledge, this study is the largest reported case series of pneumatosis intestinalis in COVID-19 patients. In our series, $77.8 \%$ of patients who were diagnosed with PI died. A majority of these patients also developed significant morbidity after the diagnosis of PI, such as increased vasopressor requirements (66.7\%) and acute kidney injury (66.7\%). For those who died, average time to death after PI diagnosis was 6.4 days while their average length of stay was 20.4 days (Table 2). These findings suggest that the development of PI is a poor prognostic factor in COVID-19.

Based on pre-COVID-19 studies on predictive factors for pathologic PI, our results support that the diagnosis of PI in COVID-19 patients is of clinical significance. Multiple studies have shown that serum lactate is the most consistent predictor of clinically significant PI. One study showed that lactate levels greater that $2.0 \mathrm{mmol} / \mathrm{L}$ at time of diagnosis has been associated with over $80 \%$ mortality [4]. In our case series, this was true in $77.8 \%$ of patients (odds ratio $=21.7$ ). The presence of small bowel dilation has also been shown to be a predictor, which was present in $55.6 \%$ of our patients (odds ratio $=1.3$ ).

The pathophysiology of this process is unclear as many aspects of COVID-19 remain unknown. Recent COVID-19 autopsy studies have shown a $40 \%$ prevalence of deep venous thrombosis, presence of microthrombi in multiple organ systems, as well evidence of intestinal endothelitis suggesting both macro and microvascular clotting predisposition [7-9]. However, the majority of patients in our case series developed PI despite therapeutic anticoagulation (77.8\%), suggesting that there may be other contributing factors. The usage of opiate medications (77.8\%) and paralytics (33.3\%) with subsequent decreased gut motility can in theory increase intraluminal pressures and cause intestinal distention, increasing risk for wall injury.

While each patient had indications for emergent surgical exploration based on pre-COVID-19 studies, risks of surgical intervention were deemed to outweigh benefits for most. The only patient with surgical intervention had undergone exploratory laparotomy the same day of PI diagnosis and died the next day. Two patients (22.2\%) had resolution of PI without surgical intervention, one of which survived to discharge. It is unclear if early surgical intervention would be beneficial.

This manifestation of disease has significant implications on possible treatment modalities of interest. Recently, "cytokine storm" has been purported to be partly responsible for the high morbidity and mortality seen in COVID-19. Interleukin-6 inhibitors are being investigated for their potential in stemming hyperinflammation and its deleterious effects. Tocilizumab, an Il-6 inhibitor, has a known elevated risk of intestinal perforation $[10,11]$. Intestinal perforation has also been reported with sarilumab, another inhibitor. In our group, five patients (55.6\%) had received an IL-6 inhibitor with an average time from administration to PI diagnosis of 11.4 days. Of these five patients, four received tocilizumab and one received sarilumab. The effect of receiving these medications on their development of PI is unclear.

\section{Conclusions}

We describe a case series of COVID-19 patients who developed pneumatosis intestinalis. We believe it is a clinically significant manifestation of disease that is likely underdiagnosed. Clinical suspicion should be high in COVID-19 patients with clinical decompensation, rising serum lactate, or worsening abdominal exam. Further surgical and pathology data is necessary to determine exact pathophysiology.

\section{Additional Information \\ Disclosures}

Human subjects: Consent was obtained by all participants in this study. Northwell Institutional Review 
Board issued approval IRB number 20-0200. Animal subjects: All authors have confirmed that this study did not involve animal subjects or tissue. Conflicts of interest: In compliance with the ICMJE uniform disclosure form, all authors declare the following: Payment/services info: All authors have declared that no financial support was received from any organization for the submitted work. Financial relationships: All authors have declared that they have no financial relationships at present or within the previous three years with any organizations that might have an interest in the submitted work. Other relationships: All authors have declared that there are no other relationships or activities that could appear to have influenced the submitted work.

\section{References}

1. Mehmood A, Essrani R, Parvataneni S, Iqbal U: Unique case of gastroenteritis presenting as pneumatosis intestinalis with emphysematous gastritis and portal vein gas: do not take gastroenteritis lightly. Cureus. 2020, 12 :e8765. 10.7759/cureus.8765

2. Gupta AK, Vazquez OA, Lopez-Viego M: Idiopathic pneumatosis of small bowel and bladder . Cureus. 2020, 12:e8313. 10.7759/cureus.8313

3. Umapathi BA, Friel CM, Stukenborg GJ, Hedrick TL: Estimating the risk of bowel ischemia requiring surgery in patients with tomographic evidence of pneumatosis intestinalis. Am J Surg. 2016, 212:762-768. 10.1016/j.amjsurg.2015.09.010

4. Hawn MT, Canon CL, Lockhart ME, et al.: Serum lactic acid determines the outcomes of CT diagnosis of pneumatosis of the gastrointestinal tract. Am Surg. 2004, 70:19-24.

5. DuBose JJ, Lissauer M, Maung AA, et al.: Pneumatosis Intestinalis Predictive Evaluation Study (PIPES): a multicenter epidemiologic study of the Eastern Association for the Surgery of Trauma. J Trauma Acute Care Surg. 2013, 75:15-23. 10.1097/TA.0b013e318298486e

6. Bhayana R, Som A, Li MD, et al.: Abdominal imaging findings in COVID- 19: preliminary observations . Radiology. 2020, 297:10.1148/radiol.2020201908

7. Edler C, Schröder AS, Aepfelbacher M, et al.: Dying with SARS-CoV-2 infection-an autopsy study of the first consecutive 80 cases in Hamburg, Germany. Int J Legal Med. 2020, 134:1275-1284. 10.1007/s00414-02002317-w

8. Bryce C, Grimes Z, Pujadas E, et al.: Pathophysiology of SARS-Cov- 2: targeting of endothelial cells renders a complex disease with thrombotic microangiopathy and aberrant immune response. The Mount Sinai COVID-19 autopsy experience [Preprint ahead of publication]. Med Rxiv. 2020, 10.1101/2020.05.18.20099960

9. Varga Z, Flammer AJ, Steiger P, et al.: Endothelial cell infection and endotheliitis in COVID-19. Lancet. 2020, 395:1417-1418. 10.1016/S0140-6736(20)30937-5

10. Strangfeld A, Richter A, Siegmund B, et al.: Risk for lower intestinal perforations in patients with rheumatoid arthritis treated with tocilizumab in comparison to treatment with other biologic or conventional synthetic DMARDs. Ann Rheum Dis. 2017, 76:504-510. 10.1136/annrheumdis-2016-209773

11. Schiff MH, Kremer JM, Jahreis A, Vernon E, Isaacs JD, van Vollenhoven RF: Integrated safety in tocilizumab clinical trials. Arthritis Res Ther. 2011, 13:141. 10.1186/ar3455 